\title{
Homophily in the adoption of digital proximity tracing apps shapes the evolution of epidemics
}

\author{
Giulio Burgio $\odot,{ }^{1}$ Benjamin Steinegger $\odot,{ }^{1}$ Giacomo Rapisardi $\odot,{ }^{1,2}$ and Alex Arenas $\odot^{1, *}$ \\ ${ }^{1}$ Departament d'Enginyeria Informàtica i Matemàtiques, Universitat Rovira i Virgili, 43007 Tarragona, Spain \\ ${ }^{2}$ Barcelona Supercomputing Center (BSC), Barcelona, Spain
}

(Received 18 March 2021; revised 7 May 2021; accepted 18 July 2021; published 6 August 2021)

\begin{abstract}
We study how homophily of human physical interactions affects the impact of digital proximity tracing on the epidemic evolution. Analytical and numerical results show the existence of different dynamical regimes with respect to the mixing rate between adopters and nonadopters, revealing a rich phenomenology in terms of the reproduction number as well as the attack rate. We corroborate our findings with Monte Carlo simulations on different real contact networks. Our results indicate that depending on infectivity and adoption, mixing between adopters can be beneficial as well as detrimental for disease control.
\end{abstract}

DOI: 10.1103/PhysRevResearch.3.033128

Preventing disease outbreaks is one of the greatest challenges humanity has faced in its history [1]. One of the techniques employed to fight epidemics is identifying and eventually testing or isolating the contacts of infected individuals, which is generally referred to as contact tracing [2]. In the past, contact tracing was employed to combat the spread of smallpox, tuberculosis, human immunodeficiency virus (HIV), or Ebola [3-6], to name a few. Correspondingly, great efforts have been devoted to analyze the efficacy of manual contact tracing [7-16]. More recently, the advent of digital proximity tracing (DPT) apps, and their successful largescale implementation to prevent the spread of severe acute respiratory syndrome coronavirus 2 (SARS-COV-2) [17-20], sparked numerous studies that analyzed how this novel technology impacts disease propagation. Many of these studies were tailored for SARS-COV-2 and quantified the impact of DPT apps [21-29]. However, also a variety of more theoretical studies unveiled the physics behind DPT [30-33].

A question which has yet to be addressed is how homophilic adoption between individuals affects the efficacy of DPT. Empirical studies suggest that the voluntary adoption of DPT apps strongly varies among the population. It was shown to correlate with age, income, and nationality [29,34,35]. Adoption is particularly low among socioeconomically marginalized classes [34]. Accordingly, due to the similarity of social contacts, usually referred to as homophily [36], app adoption is much more probable for contacts of an app user (adopter) compared to a random individual. As a matter of fact, in Switzerland around $70 \%$ of the contacts among adopters were found to use the app as well, while on average, national adoption is only $20 \%$ [19]. This discrep-

\footnotetext{
*alexandre.arenas@urv.cat

Published by the American Physical Society under the terms of the Creative Commons Attribution 4.0 International license. Further distribution of this work must maintain attribution to the author(s) and the published article's title, journal citation, and DOI.
}

ancy is in line with experimental studies that indicate how homophily importantly affects health behavior [37,38]. Such evidence lets us theorize that this is another manifestation of how human behavior crucially shapes the course of epidemics $[39,40]$.

Our goal here is to follow this hypothesis and unveil how the homophilic adoption of DPT apps affects disease propagation. For this aim, we extend a model recently introduced by Bianconi et al. [31]. The model is very convenient due to its simplicity, while it captures the essential ingredients of the dynamics. As a first step, we consider a mean-field case in which we control the interaction rate among adopters and nonadopters. This simple setup shows different regimes in which the homophilic adoption can be beneficial as well as detrimental. Furthermore, we analytically show how the reproduction number, i.e., the number of secondary infections caused by an infected individual, is minimized for a specific value of homophily. In a second step, we analyze the impact of homophily on a real-world primary-school network. The network exhibits the same dynamical regimes and thus underpins the theoretical results.

To prove our hypothesis, we consider the standard susceptible-infected-recovered (SIR) model, with transmission probability $\lambda$, infectious period $\tau$, and contact rate $k$. For convenience we define $\beta=\lambda \tau$. Accordingly, in the absence of an intervention, i.e., app adoption, and assuming homogeneous mixing, the basic reproduction number of the disease is given by $R_{0}=\beta k$. We fix the fraction of adopters as $T \in[0,1]$. Furthermore, we parametrize the mixing relation between adopters and nonadopters, i.e., the contact matrix $K$ (often referred to as the who-acquires-infection-from-whom matrix [2]), with a parameter $\alpha \in[0,1]$. We denote the entries of $K$ as $k_{i j}$ with $i, j \in\{\mathrm{A}, \mathrm{N}\}$, where $\mathrm{A}$ and $\mathrm{N}$ refer to adopters and nonadopters, respectively. The parameter $\alpha$ fixes the contact rate between adopters and nonadopters as $k_{\mathrm{AN}}=\alpha k(1-T)$. In other words, $\alpha$ interpolates from complete homophily ( $\alpha=0)$ to random mixing ( $\alpha=1$ ).

This parametrization does not allow for disassortativity. However, since the empirical evidence clearly indicates a 
positive correlation between social contacts and app adoption, we disregard this possibility. Eventually, the remaining contact rates follow from the balance equation $T k_{\mathrm{AN}}=$ $(1-T) k_{\mathrm{NA}}$ and the average contact rate $k=k_{\mathrm{AA}}+k_{\mathrm{AN}}=$ $k_{\mathrm{NN}}+k_{\mathrm{NA}}$. Accordingly, $K$ has the following entries,

$$
\begin{gathered}
k_{\mathrm{AN}}=\alpha(1-T) k, \\
k_{\mathrm{NA}}=\alpha T k, \\
k_{\mathrm{AA}}=[1-\alpha(1-T)] k, \\
k_{\mathrm{NN}}=[1-\alpha T] k .
\end{gathered}
$$

With these definitions, the adoption homophily $h$, i.e., the probability that during a contact both individuals are either adopters or not, reads

$$
h=\frac{1}{k}\left[T k_{\mathrm{AA}}+(1-T) k_{\mathrm{NN}}\right]=1-2 \alpha T(1-T) .
$$

The dynamical model introduced by Bianconi et al. [31] is based on the assumption that app users infected by other app users do not further transmit the disease. This assumption requires us to define additional variables to follow the dynamical evolution in comparison to the standard SIR model. Namely, we define $I_{\mathrm{AA}}(t)$ and $I_{\mathrm{AN}}(t)$ as the number of newly infected (incidence) adopters at generation $t$, which were infected by adopters and nonadopters, respectively. For nonadopters such a distinction is not necessary, therefore $I_{\mathrm{N}}(t)$ simply refers to the newly infected nonadopters at generation $t$. Together with the number of susceptible adopters $S_{\mathrm{A}}(t)$ and nonadopters $S_{\mathrm{N}}(t)$ at generation $t$, the discrete dynamical equations then read

$$
\begin{gathered}
I_{\mathrm{N}}(t+1)=\beta\left[k_{\mathrm{NN}} I_{\mathrm{N}}(t)+k_{\mathrm{AN}} I_{\mathrm{AN}}(t)\right] \frac{S_{\mathrm{N}}(t)}{N_{\mathrm{N}}}, \\
I_{\mathrm{AN}}(t+1)=\beta k_{\mathrm{NA}} I_{\mathrm{N}}(t) \frac{S_{\mathrm{A}}(t)}{N_{\mathrm{A}}}, \\
I_{\mathrm{AA}}(t+1)=\beta k_{\mathrm{AA}} I_{\mathrm{AN}}(t) \frac{S_{\mathrm{A}}(t)}{N_{\mathrm{A}}}, \\
S_{\mathrm{N}}(t+1)=S_{\mathrm{N}}(t)-I_{\mathrm{N}}(t+1), \\
S_{\mathrm{A}}(t+1)=S_{\mathrm{A}}(t)-I_{\mathrm{AA}}(t+1)-I_{\mathrm{AN}}(t+1) .
\end{gathered}
$$

where $N_{\mathrm{A}}$ and $N_{\mathrm{N}}$ indicate the number of adopters and nonadopters, respectively, in the population. The final attack rate, i.e., the number of recovered individuals, is found by summing the number of newly infected (then recovered) individuals over all generations.

As the classical SIR model, this dynamical system does not allow for an explicit solution of the nontrivial, stationary state. However, the model allows us to calculate the reproduction number $R$ after app adoption (intervention) but in the absence of preexisting immunity. For $R>1$, the disease initially invades the population whereas for $R<1$ it immediately dies out. The reproduction number can be calculated through the next-generation matrix $[41,42](\mathrm{NGM})$ - here, equivalent to the Jacobian of the dynamics evaluated in the disease free equilibrium — which is given by

$$
\mathrm{NGM}=\beta\left(\begin{array}{ccc}
k_{\mathrm{NN}} & k_{\mathrm{AN}} & 0 \\
k_{\mathrm{NA}} & 0 & 0 \\
0 & k_{\mathrm{AA}} & 0
\end{array}\right) .
$$

The spectral radius of NGM represents the effective reproduction number $R$. By inserting the explicit expressions of the $K$ matrix entries, one gets

$$
R=\frac{R_{0}}{2}\left[1-\alpha T+\sqrt{(1-\alpha T)^{2}+4 \alpha^{2} T(1-T)}\right] .
$$

Not surprisingly, $R$ has a monotonous dependence on adoption $T$ as well as on the basic reproduction number of the disease $R_{0}$. However, $R$ exhibits a nontrivial dependence on the mixing rate $\alpha$. Precisely, solving $\frac{d R}{d \alpha}=0$ with respect to $\alpha$ yields

$$
\alpha^{*}=1-\frac{\frac{2}{3}-T}{\frac{4}{3}-T} .
$$

Straightforward calculations also show that $\left.\frac{d R}{d \alpha}\right|_{\alpha=0}<0$ is always met. Accordingly, whenever $\alpha^{*} \geqslant 1$, the reproduction number has its smallest value at $\alpha=1$. The condition $\alpha^{*}<1$ leads to a critical value $T^{*}=2 / 3$ above which no local minimum exists. Figure 1(a) shows the reproduction number $R$ as a function of $\alpha$ for low adoption $\left(T<T^{*}\right)$. The dependence of $\alpha^{*}$ on $T$ is also indicated.

This nonmonotonous dependence on the mixing rate $\alpha$ distinguishes contact tracing from classical immunization problems such as vaccination [43]. Assuming perfect immunization, all the entries of the NGM will be zero, except the one among not immunized individuals (nonadopters). Accordingly, the dependence on $\alpha$ will be monotonous and mixing will always reduce the reproduction number.

Furthermore, Eq. (12) provides a critical parameter range in which eradication is possible, i.e., $R<1$. Since our focus is the effect of homophily, we express the condition for eradication as a function of $\alpha$. In other words, for any $\alpha \in\left(\alpha_{c}^{-}, \alpha_{c}^{+}\right)$, we have $R<1$, where

$$
\alpha_{c}^{ \pm}=\frac{1}{2 R_{0}(1-T)}\left[1 \pm \sqrt{1-4 \frac{1-T}{T}\left(R_{0}-1\right)}\right] .
$$

The existence of two physical solutions of $\alpha_{c}$ implies that increasing mixing may not only push the system below threshold, but also push it above for $\alpha>\alpha_{c}^{+}$, and thus hinder eradication. This possibility emerges from the existence of a local minimum in the reproduction number with respect to the mixing rate $\alpha$.

Figure 1(b) shows the attack rate as a function of $\alpha$ and the basic reproduction number $R_{0}$. The solid line indicates $\alpha_{c}^{-}$. While the attack rate monotonously increases with $R_{0}$, the latter separates three different dynamical regimes regarding the dependence of the attack rate on $\alpha$, as indicated by the dashed lines.

In Fig. 1(c) we escrutinize these three regimes by presenting the attack rate as a function of $\alpha$ following the dashed lines. We categorize these regimes, in ascending order with respect to $R_{0}$, as critical, intermediate, and saturated. Close to threshold (critical), the attack rate decreases with the mixing between adopters and nonadopters. In the intermediate 

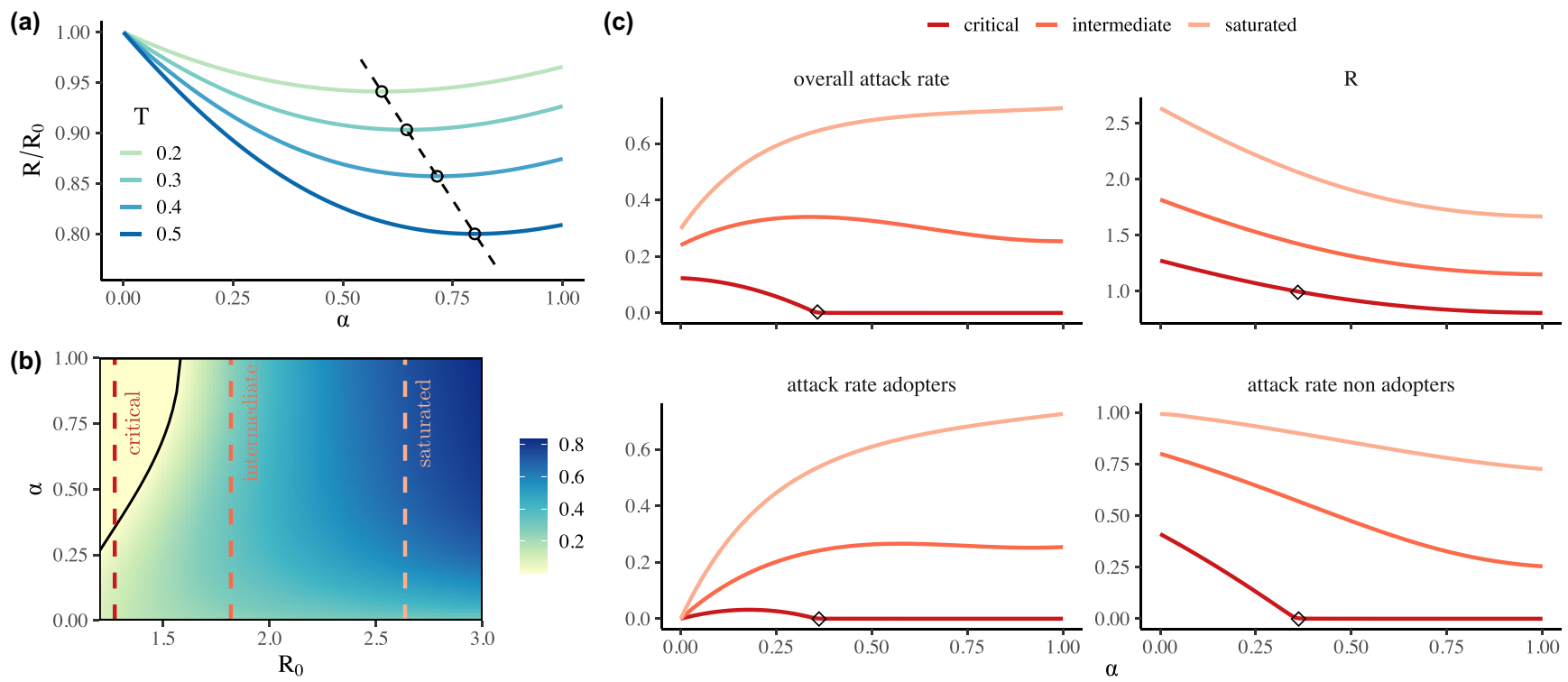

FIG. 1. (a) Reproduction number $R$ normalized with respect to the basic reproduction number $R_{0}$ for different values of adoption $T$ below $T^{*}$. Dots indicate the minimum at $\alpha^{*}$, while the dashed line shows its variation for $T \in[0.1,0.55]$. (b) Numerical solution of the dynamics for the attack rate as a function of $\alpha$ and $R_{0}$. The solid line indicates the threshold $\alpha_{c}^{-}$for which $R=1$. Colored, dashed lines denote the dynamical regimes: critical, intermediate, and saturated. Adoption was fixed as $T=0.7$. For these parameter values we have $\alpha_{c}^{+}>1$. (c) Top panels show the attack rate and the reproduction number for the different regimes defined in (b). The specific attack rates for adopters and nonadopters are reported in the bottom panels. Black diamonds indicate $\alpha_{c}^{-}$, at which $R=1$.

regime, instead, we observe a nonmonotonous dependence of the attack rate on $\alpha$. Finally, far from the epidemic threshold, in a saturated regime, the attack rate always increases with $\alpha$.

The variety of regimes stems from the competition between two processes. On the one hand, as mixing increases, adopters provide protection to nonadopters. This is illustrated by a decreasing reproduction number $R$ [top-right panel in Fig. 1(c)] and attack rate among nonadopters [bottom-right panel in Fig. 1(c)]. On the other hand, protection vanishes inside the adoption cluster [bottom-left panel in Fig. 1(c)]. Adoption and infectivity, i.e., the basic reproduction number $R_{0}$, then determine which of these two processes holds the upper hand. For low coverage, complete, homophilic adoption is more beneficial, since random mixing does not provide any protection-neither to nonadopters nor to adopters. In contrast, for high coverage, a random distribution of adopters acts as a firewall for nonadopters and enables us to immunize the population. The same interplay causes the minimum at $\alpha=\alpha^{*}$ in the reproduction number $R$. As illustrated in Fig. 1(a), the lower the adoption coverage, the more the homophily (lower $\alpha^{*}$ ), i.e., the clustered protection, necessary to minimize $R$.

However, whether a given adoption is sufficient to prevent the infection of nonadopters is determined by the basic reproduction number $R_{0}$. Far from the critical threshold, in the saturated regime, the attack rate among nonadopters only slowly varies with the reproduction number $R$. Accordingly, mixing increases the attack rate. In contrast, close to the threshold, in the critical regime, the attack rate among nonadopters strongly varies with a decrease in the reproduction number. Therefore, mixing is beneficial and allows us to push the system below the critical point, from which then also adopters benefit. Finally, in the intermediate regime, the system switches between critical and saturated, wherefore we observe a nonmonotonous dependence with respect to $\alpha$.

We kept the coverage of the app $T$ fixed in the above analysis of the different regimes. To investigate the effect of coverage, we fix the basic reproduction number $R_{0}$ and vary $\alpha$ for different values of $T$. Figure 2 shows the attack rate as a function of the reproduction number $R$. While colors indicate different values of $T$, the mixing rate $\alpha$ is mapped to point size. For high values of $T(0.9$ and 0.7$)$, the system is in between the intermediate and critical regime. Accordingly, we find a local maximum, but the disease can also be eradicated, $R<1$. For lower values of $T(0.5$ and 0.3$)$, we observe a local minimum of the reproduction number since $T<T^{*}$. This

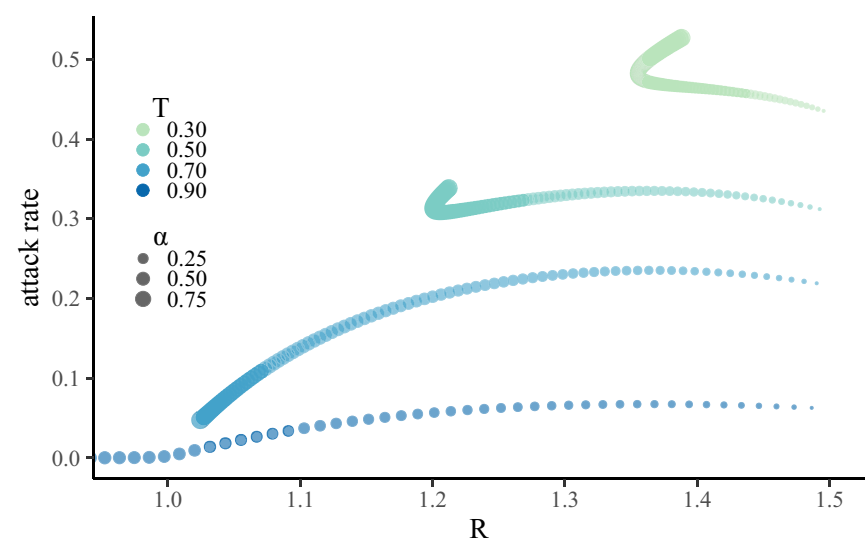

FIG. 2. Attack rate as a function of the reproduction number $R$ for different values of adoption $T$. The size of the points interpolates between $\alpha=0$ and $\alpha=1$. We fixed the basic reproduction number as $R_{0}=1.5$. 

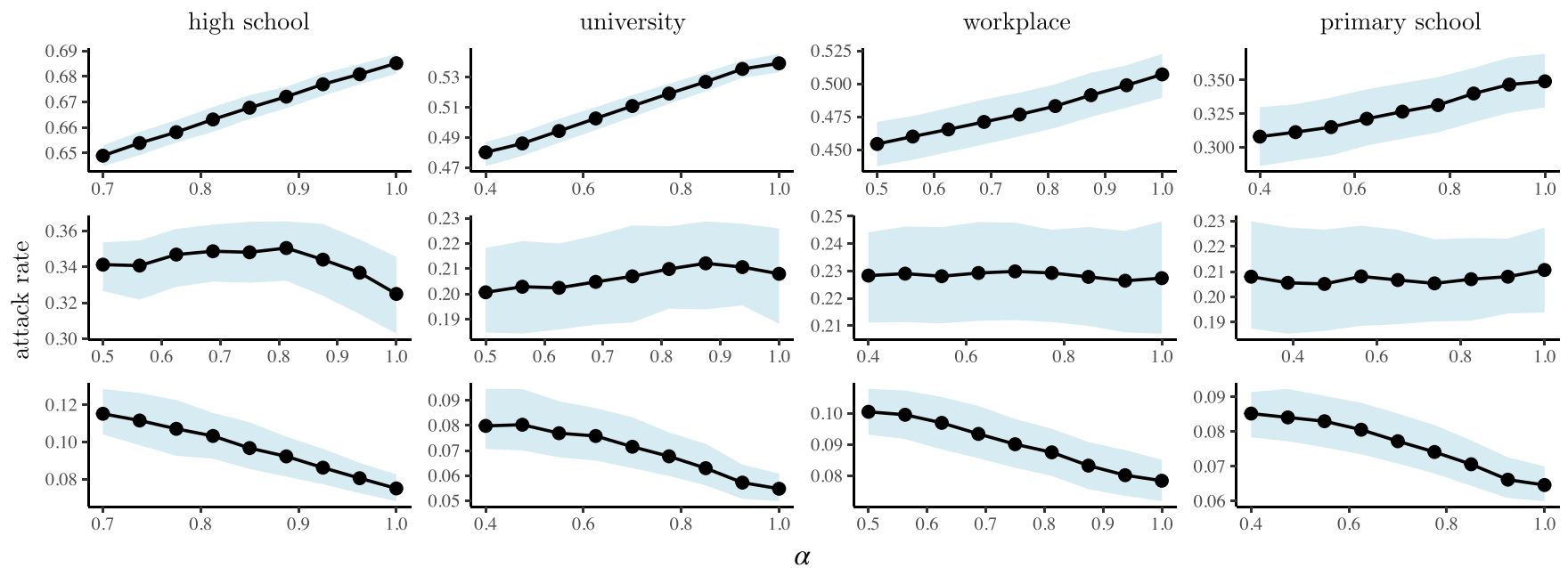

FIG. 3. Dependence of the attack rate on $\alpha$ as resulting from Monte Carlo simulations for the three dynamical regimes and four different real-world networks. Each column refers to a different network, while rows indicate the critical, intermediate, and saturated regime, respectively, from top to bottom. Note that different values of $\alpha$ can be accessed depending on adoption $T$ and structural constraints of the network. Dots indicate the median value, whereas the ribbon indicates first and third quartiles. Each point is obtained by averaging over $4 \times 10^{4}$ runs. From left to right, the networks have 780, 636, 212, and 226 nodes. For the saturated and critical regimes we fixed $T=0.30$ and $T=0.90$, respectively. In the intermediate regime, $T$ was fixed as $0.75,0.72,0.67$, and 0.69 for the different networks (left to right). Specific to each network we set $\beta$ as $0.030,0.085,0.110$, and 0.090 .

local minimum in the reproduction number then causes an additional minimum in the attack rate for $T=0.5$. In contrast, for $T=0.3$ the attack rate only increases with $\alpha$. Notably, due to the nonmonotonous form of $R$, we observe different attack rates for equal values of the reproduction number. Overall, Fig. 2 illustrated how the three dynamical regimes can also be reached by varying adoption $T$.

We corroborate our theory through Monte Carlo simulations on a diverse set of real-world contact networks [44-47]. To transform the temporal networks into static, binary ones, we threshold the aggregated weights. We distribute apps algorithmically to control the value of $\alpha$, which is calculated through Eq. (5). The algorithm starts with a random distribution of apps $(\alpha \approx 1)$. Then, until the desired value of $\alpha$ is reached (tolerance of 0.02 ), the roles of a randomly chosen adopter and nonadopter are interchanged whenever this leads to a decrease in $\alpha$. By checking the average degree of adopters and nonadopters, we guarantee that no correlation is created between app adoption and number of contacts. Figure 3 reports the results of Monte Carlo simulations for the attack rate versus $\alpha$. The curves, each corresponding to a different value of $T$, show the nontrivial phenomenological change through the three dynamical regimes of the epidemics, i.e., saturated, intermediate, and critical, as correctly identified by our analytical model. For the smallest networks-workplace and high school-the little dependence on $\alpha$ found for the intermediate regime in the analytical model is likely to be hidden by the stochastic fluctuations. However, despite their small size, one can clearly distinguish three different regimes.

To sum up, we analyzed how the homophilic adoption of DPT apps affects the disease dynamics. We unveiled the existence of different dynamical regimes, originating from a nontrivial dependence on the mixing rate. In the critical regime, mixing is beneficial, may enable to push the system below threshold, and thus eradicate the disease. Far from the threshold, mixing is detrimental due to a waning protection among app users. Finally, for an intermediate case, the system switches between the two regimes with varying mixing rate, and the dependence is nonmonotonous. Moreover, we discovered a local minimum in the reproduction number, existing whenever adoption is smaller than $2 / 3$. Accordingly, an increasing mixing rate may even push the system above the critical threshold and cause the disease to spread. Interestingly, the different regimes in the attack rate can arise independently on whether the reproduction has a local minimum or not.

We verified that the results are robust against the introduction of imperfections in notification and isolation (see Supplemental Material [48]). In this sense, the key ingredient for the physics of DPT is the necessity that during a contact both are adopters in order to reduce further transmission. Additionally, the phenomenology remains unchanged, except for a rescaling of $R_{0}$, for additional heterogeneities in the population as long as they do not correlate with app adoption. At the cost of model tractability, the framework presented here may be extended to analyze the role of possible correlations with, for example, the reduction of contacts (social distancing) or the adoption of other prophylactic measures (face masks). In particular, if the aim was to empirically quantify the impact of homophily, such extensions as well as to explicitly account for the temporal nature of social contacts would be necessary.

Switching our focus to the real world, the adoption of DPT apps is generally very low, between $20 \%$ and $40 \%[19,20]$. Accordingly, it is very improbable to control the epidemic with such low adoption. In this sense, our results indicate that homophilic adoption is beneficial to this point. However, if health authorities desire to actually contain the spread of SARS-COV-2 through large-scale adoption, i.e., reach the critical regime, overcoming homophily in health behavior-in 
particular, with respect to different socioeconomic classeswill be crucial.

G.B. acknowledges financial support from the European Union's Horizon 2020 research and innovation program under the Marie Skłodowska-Curie Grant Agreement No. 945413 and from the Universitat Rovira i Virgili (URV). B.S. acknowledges financial support from the European Unions Horizon 2020 research and innovation program under the Marie Skłodowska-Curie Grant Agreement No. 713679 and from the
Universitat Rovira i Virgili (URV). A.A. acknowledges support by Ministerio de Economía y Competitividad (Grants No. PGC2018-094754-B-C21 and No. FIS2015-71582-C2-1), Generalitat de Catalunya (Grants No. 2017SGR-896 and No. 2020PANDE00098), Universitat Rovira i Virgili (Grant No. 2019PFR-URV-B2-41), ICREA Academia, and the James S. McDonnell Foundation (Grant No. 220020325). We thank L. Arola-Fernández and A. Cardillo for helpful comments and suggestions.

G.B. and B.S. contributed equally to this work.
[1] W. H. McNeill, Plagues and Peoples (Anchor Books, New York, 1976).

[2] P. Rohani and M. Keeling, Modeling Infectious Diseases in Humans and Animals (Princeton University Press, Princeton, NJ, 2011).

[3] W. H. Foege, J. D. Millar, and J. M. Lane, Selective epidemiologic control in smallpox eradication, Am. J. Epidemiol. 94, 311 (1971).

[4] G. J. Fox, S. E. Barry, W. J. Britton, and G. B. Marks, Contact investigation for tuberculosis: A systematic review and metaanalysis, Eur. Respir. J. 41, 140 (2013).

[5] G. W. Rutherford and J. M. Woo, Contact tracing and the control of human immunodeficiency virus infection, J. Am. Med. Assoc. 259, 3609 (1988).

[6] K. C. Swanson, C. Altare, C. S. Wesseh, T. Nyenswah, T. Ahmed, N. Eyal, E. L. Hamblion, J. Lessler, D. H. Peters, and M. Altmann, Contact tracing performance during the Ebola epidemic in Liberia, 2014-2015, PLoS Neglected Trop. Dis. 12, e0006762 (2018).

[7] M. R. Howell, W. J. Kassler, and A. Haddix, Partner notification to prevent pelvic inflammatory disease in women, Sex. Transm. Dis. 24, 287 (1997).

[8] M. Kretzschmar, Y. T. Van Duynhoven, and A. J. Severijnen, Modeling prevention strategies for gonorrhea and chlamydia using stochastic network simulations, Am. J. Epidemiol. 144, 306 (1996)

[9] C. Fraser, S. Riley, R. M. Anderson, and N. M. Ferguson, Factors that make an infectious disease outbreak controllable, Proc. Natl. Acad. Sci. USA 101, 6146 (2004).

[10] D. Klinkenberg, C. Fraser, and H. Heesterbeek, The effectiveness of contact tracing in emerging epidemics, PLoS ONE 1, e12 (2006).

[11] T. C. Porco, K. A. Holbrook, S. E. Fernyak, D. L. Portnoy, R. Reiter, and T. J. Aragón, Logistics of community smallpox control through contact tracing and ring vaccination: A stochastic network model, BMC Public Health 4, 34 (2004).

[12] J. Müller, M. Kretzschmar, and K. Dietz, Contact tracing in stochastic and deterministic epidemic models, Math. Biosci. 164, 39 (2000).

[13] I. Z. Kiss, D. M. Green, and R. R. Kao, Infectious disease control using contact tracing in random and scale-free networks, J. R. Soc. Interface 3, 55 (2006).

[14] K. T. Eames and M. J. Keeling, Contact tracing and disease control, Proc. R. Soc. B 270, 2565 (2003).

[15] M. Eichner, Case isolation and contact tracing can prevent the spread of smallpox, Am. J. Epidemiol. 158, 118 (2003).
[16] N. G. Becker, K. Glass, Z. Li, and G. K. Aldis, Controlling emerging infectious diseases like SARS, Math. Biosci. 193, 205 (2005).

[17] P. Rodríguez, S. Graña, E. E. Alvarez-León, M. Battaglini, F. J. Darias, M. A. Hernán, R. López, P. Llaneza, M. C. Martín, O. Ramirez-Rubio, A. Romaní, B. Suárez-Rodríguez, J. Sánchez-Monedero, A. Arenas, and L. Lacasa, A populationbased controlled experiment assessing the epidemiological impact of digital contact tracing, Nat. Commun. 12, 587 (2021).

[18] I. Braithwaite, T. Callender, M. Bullock, and R. W. Aldridge, Automated and partly automated contact tracing: A systematic review to inform the control of COVID-19, Lancet Digital Health 2, E607 (2020).

[19] M. Salathé, C. L. Althaus, N. Anderegg, D. Antonioli, T. Ballouz, E. Bugnion, S. Capkun, D. Jackson, S.-I. Kim, J. Larus et al., Early evidence of effectiveness of digital contact tracing for SARS-COV-2 in Switzerland, Swiss Med. Wkly. 150, w20457 (2020).

[20] C. Wymant, L. Ferretti, D. Tsallis, M. Charalambides, L. Aberler-Dörner, D. Bonsall, R. Hinch, M. Kendall, L. Milson, M. Ayres, C. Holmes, M. Briers, and C. Fraser, The epidemiological impact of the NHS COVID-19 app, Nature 594, 408 (2021).

[21] A. J. Kucharski, P. Klepac, A. J. Conlan, S. M. Kissler, M. L. Tang, H. Fry, J. R. Gog, W. J. Edmunds, J. C. Emery, G. Medley et al., Effectiveness of isolation, testing, contact tracing, and physical distancing on reducing transmission of SARS-COV-2 in different settings: A mathematical modelling study, Lancet Infect. Dis. 20, 1151 (2020).

[22] W. J. Bradshaw, E. C. Alley, J. H. Huggins, A. L. Lloyd, and K. M. Esvelt, Bidirectional contact tracing could dramatically improve COVID-19 control, Nat. Commun. 12, 232 (2021).

[23] A. Aleta, D. Martin-Corral, A. P. y Piontti, M. Ajelli, M. Litvinova, M. Chinazzi, N. E. Dean, M. E. Halloran, I. M. Longini, Jr., S. Merler et al., Modelling the impact of testing, contact tracing and household quarantine on second waves of COVID-19, Nat. Hum. Behav. 4, 964 (2020).

[24] L. Ferretti, C. Wymant, M. Kendall, L. Zhao, A. Nurtay, L. Abeler-Dörner, M. Parker, D. Bonsall, and C. Fraser, Quantifying SARS-CoV-2 transmission suggests epidemic control with digital contact tracing, Science 368, eabb6936 (2020).

[25] A. Barrat, C. Cattuto, M. Kivelä, S. Lehmann, and J. Saramäki, Effect of manual and digital contact tracing on COVID-19 outbreaks: A study on empirical contact data, J. R. Soc. Interface 18, 20201000 (2021). 
[26] J. Hellewell, S. Abbott, A. Gimma, N. I. Bosse, C. I. Jarvis, T. W. Russell, J. D. Munday, A. J. Kucharski, W. J. Edmunds, F. Sun, S. Flasche, B. J. Quilty, N. Davies, Y. Liu, S. Clifford, P. Klepac, M. Jit, C. Diamond, H. Gibbs, K. van Zandvoort, S. Funk, and R. M. Eggo, Feasibility of controlling COVID19 outbreaks by isolation of cases and contacts, Lancet Global Health 8, e488 (2020).

[27] L. Lorch, H. Kremer, W. Trouleau, S. Tsirtsis, A. Szanto, B. Schölkopf, and M. Gomez-Rodriguez, Quantifying the effects of contact tracing, testing, and containment measures in the presence of infection hotspots, arXiv:2004.07641.

[28] G. Cencetti, G. Santin, A. Longa, E. Pigani, A. Barrat, C. Cattuto, S. Lehmann, M. Salathé, and B. Lepri, Digital proximity tracing on empirical contact networks for pandemic control, Nat. Commun. 12, 1655 (2021).

[29] J. A. Moreno López, B. A. García, P. Bentkowski, L. Bioglio, F. Pinotti, P.-Y. Boëlle, A. Barrat, V. Colizza, and C. Poletto, Anatomy of digital contact tracing: Role of age, transmission setting, adoption and case detection, Sci. Adv. 7, eabd8750 (2021).

[30] A. Reyna-Lara, D. Soriano-Paños, S. Gómez, C. Granell, J. T. Matamalas, B. Steinegger, A. Arenas, and J. Gómez-Gardeñes, Virus spread versus contact tracing: Two competing contagion processes, Phys. Rev. Research 3, 013163 (2021).

[31] G. Bianconi, H. Sun, G. Rapisardi, and A. Arenas, Messagepassing approach to epidemic tracing and mitigation with apps, Phys. Rev. Research 3, L012014 (2021).

[32] I. Kryven and C. Stegehuis, Contact tracing in configuration models, J. Phys.: Complexity 2, 025004 (2021).

[33] S. Kojaku, L. Hébert-Dufresne, E. Mones, S. Lehmann, and Y.-Y. Ahn, The effectiveness of backward contact tracing in networks, Nat. Phys. 17, 652 (2021).

[34] V. von Wyl, M. Höglinger, C. Sieber, M. Kaufmann, A. Moser, M. Serra-Burriel, T. Ballouz, D. Menges, A. Frei, and M. A. Puhan, Drivers of acceptance of COVID-19 proximity tracing apps in Switzerland: Panel survey analysis, JMIR Public Health Surveill. 7, e25701 (2021).

[35] S. Munzert, P. Selb, A. Gohdes, L. F. Stoetzer, and W. Lowe, Tracking and promoting the usage of a COVID-19 contact tracing app, Nat. Hum. Behav. 5, 247 (2021).
[36] M. McPherson, L. Smith-Lovin, and J. M. Cook, Birds of a feather: Homophily in social networks, Annu. Rev. Sociol. 27, 415 (2001).

[37] D. Centola, The spread of behavior in an online social network experiment, Science 329, 1194 (2010).

[38] D. Centola, An experimental study of homophily in the adoption of health behavior, Science 334, 1269 (2011).

[39] S. Funk, M. Salathé, and V. A. A. Jansen, Modelling the influence of human behaviour on the spread of infectious diseases: A review, J. R. Soc. Interface 7, 1247 (2010).

[40] Z. Wang, C. T. Bauch, S. Bhattacharyya, A. d'Onofrio, P. Manfredi, M. Perc, N. Perra, M. Salathé, and D. Zhao, Statistical physics of vaccination, Phys. Rep. 664, 1 (2016).

[41] O. Diekmann, J. A. Heesterbeek, and J. A. Metz, On the definition and the computation of the basic reproduction ratio $R_{0}$ in models for infectious diseases in heterogeneous populations, J. Math. Biol. 28, 365 (1990).

[42] O. Diekmann, J. Heesterbeek, and M. G. Roberts, The construction of next-generation matrices for compartmental epidemic models, J. R. Soc. Interface 7, 873 (2010).

[43] M. Salathé and S. Bonhoeffer, The effect of opinion clustering on disease outbreaks, J. R. Soc. Interface 5, 1505 (2008).

[44] M. Salathé, M. Kazandjieva, J. W. Lee, P. Levis, M. W. Feldman, and J. H. Jones, A high-resolution human contact network for infectious disease transmission, Proc. Natl. Acad. Sci. USA 107, 22020 (2010).

[45] P. Sapiezynski, A. Stopczynski, D. D. Lassen, and S. Lehmann, Interaction data from the Copenhagen networks study, Sci. Data 6, 315 (2019).

[46] M. Génois and A. Barrat, Can co-location be used as a proxy for face-to-face contacts?, EPJ Data Sci. 7, 11 (2018).

[47] J. Stehlé, N. Voirin, A. Barrat, C. Cattuto, L. Isella, J.-F. Pinton, M. Quaggiotto, W. Van den Broeck, C. Régis, B. Lina et al., High-resolution measurements of face-to-face contact patterns in a primary school, PLoS ONE 6, e23176 (2011).

[48] See Supplemental Material at http://link.aps.org/supplemental/ 10.1103/PhysRevResearch.3.033128 for we present an extended analysis relaxing the hypothesis of a fully effective contact tracing app, and accounting for additional heterogeneities in the population, e.g., number of contacts. 